\title{
Kaon production from hot and dense matter formed in heavy-ion collisions
}

\author{
G. E. Brown \\ Physics Department, State University of New York at Stony Brook, Stony Brook, New York 11794 \\ C. M. Ko, Z. G. Wu, and L. H. Xia* \\ Cyclotron Institute and Physics Department, Texas A\&M University, College Station, Texas 77843
}

(Received 19 September 1990)

\begin{abstract}
In heavy-ion collisions, kaons can be produced from baryon-baryon, meson-baryon, and mesonmeson interactions. Simple meson-exchange models are introduced to study kaon production from these processes in the free space. These models are then extended to determine kaon production in hot, dense nuclear matter by taking into account the decreasing hadron masses as a result of the restoration of chiral symmetry and the condensation of kaons. We find that the cross sections for kaon production from all three processes are enhanced. In particular, the effect of decreasing hadron masses on kaon production from the meson-meson annihilation is most significant. In the hydrochemical model for heavy-ion collisions, we demonstrate that the observed enhancement of kaon yield in high-energy heavy-ion collisions can be explained if the medium effect is included.
\end{abstract}

\section{INTRODUCTION}

High-energy heavy-ion collisions offer the possibility of creating a high-density nuclear matter in the initial stage of the collision. According to transport models, the nuclear matter density can reach about $3 \rho_{0}$, where $\rho_{0}$ is the normal nuclear matter density, at the Bevalac energies of up to $2 \mathrm{GeV} /$ nucleon. $^{1}$ Moving up to the alternatinggradient synchrotron (AGS) energies of about 15 $\mathrm{GeV} /$ nucleon, the initial density is even higher and the central density exceeds ${ }^{2} 6 \rho_{0}$. The creation of such a high-density matter is naturally accompanied by high excitations. Although an equilibrium state may not be achieved due to the finite size of the colliding systems, it is helpful to use the concept of temperature to describe qualitatively the excitation. The temperature of the initial high-density matter is then about $50-100 \mathrm{MeV}$ at the Bevalac energies ${ }^{1}$ and about $150-200 \mathrm{MeV}$ at the AGS energies. $^{2}$ In such a hot, dense nuclear matter, one expects that not only the properties of hadrons but also their interactions are modified. There are already some studies on the medium effect on the nucleon-nucleon interaction. ${ }^{3-6}$ It has been found that the nucleon-nucleon cross section increases substantially with the density. Also, works have been carried out in studying dilepton production from pion-pion annihilation in heavy-ion collisions to see how the medium-modified pions affect its production rate. ${ }^{7-9}$

Because of the conservation of strangeness, kaons will not be absorbed by the nuclear matter after creation and therefore carry information on their production mechanism. At the Bevalac energies, kaon production is mainly through the process $B B \rightarrow B Y K$, where $B, Y$, and $K$ stand for baryons, hyperons, and kaons, respectively. ${ }^{10,11}$ The process $\pi B \rightarrow Y K$ from the interaction of secondary pions with baryons gives only about $25 \%$ of the total contribution while the process $\pi \pi \rightarrow K \bar{K}$ from pion-pion annihilation is negligible. ${ }^{12,13}$ Kaon production from nuclear re- actions has also been studied at the AGS energies. ${ }^{14,15}$ The measured $K^{+} / \pi^{+}$ratio is about $4 \%$ from the $p+\mathrm{Be}$ interaction and is similar to that from the proton-proton interaction at the same energy. This is consistent with the assumption that the dominant process is $B B \rightarrow B Y K$. In the $p$-Au interaction, this ratio is increased to about $10 \%$ and the peak of the kaon rapidity distribution is also shifted from the nucleon-nucleon center-of-mass rapidity towards that of the target. This implies that the contribution from the secondary process $\pi B \rightarrow Y K$ becomes important. For heavy-ion collisions such as $\mathrm{Si}+\mathrm{Au}$, this ratio increases to about $20 \%$ and strongly suggests the need for the contribution from $\pi \pi \rightarrow K \bar{K}$. On the other hand, the $K^{-} / \pi^{-}$ratio remains about $4 \%$ for both proton and nucleus induced reactions.

The enhanced kaon production can, of course, be explained if one assumes that a quark-gluon plasma is formed in the initial stage of the collision. ${ }^{16,17}$ From the analysis of the rapidity and transverse energy distributions of measured particles, the extracted energy density seems to be lower than that expected for the formation of the quark-gluon plasma. ${ }^{18,19}$

In the hadronic scenario, both thermal and transport models have been used to study kaon production in highenergy heavy-ion collisions. Using the hydrochemical model, it has been shown that, for reasonable initial density and temperature, the $K^{+} / \pi^{+}$ratio is only about $10 \%$ if a cross section of about $3 \mathrm{mb}$ is taken for the process $\pi \pi \rightarrow K \bar{K} .{ }^{16}$ In calculations based on the transport model, a similar ratio ${ }^{20}$ is obtained if $\bar{\sigma}_{\pi \pi \rightarrow K \bar{K}}$ is taken to be $0.5 \mathrm{mb}$ and a ratio comparable to the measured one is found in Ref. 21 with $\bar{\sigma}_{\pi \pi \rightarrow K \bar{K}} \sim 2-3 \mathrm{mb}$. In both transport calculations, the dominant contribution is from $B B \rightarrow B Y K$ and somewhat more pions appear to be produced by this process in Ref. 21 .

In these studies, the elementary kaon production cross sections for the two processes $B B \rightarrow B Y K$ and $\pi B \rightarrow Y K$ are extracted from empirical data ${ }^{10,12}$ and should be quite 
reliable. The process $\pi \pi \rightarrow K \bar{K}$ has recently analyzed in detail by Lohse et al. ${ }^{22}$ and compared with results from Protopopescu et al.$^{23}$ and phase shifts from Martin and Ozmutlu. ${ }^{24}$ The cross section is appreciable only in the isospin-zero channel, where $\sigma(I=0) \approx 9 \mathrm{mb}$ in the region the center-of-mass energy $s^{1 / 2}=1-1.4 \mathrm{GeV}$. Isospin averaging introduces, therefore, a factor of $\frac{1}{9}$, so that the averaged cross section is $\bar{\sigma}_{\pi \pi \rightarrow K \bar{K}} \sim 1 \mathrm{mb}$. The cross section used in Refs. 16, 20, and 21 are thus not unreasonable.

In previous studies, the effect of hot, dense nuclear matter on kaon production has been ignored. Kaplan and Nelson ${ }^{25}$ noted that chiral Lagrangians with empirical determined coefficients automatically contain a kaon mass term that decreases with increasing density. Typical pion energies in the E802 experiment at the AGS are about that of the free kaon mass so that there is little phase space for the reaction $\pi \pi \rightarrow K \bar{K}$. The phase space increases rapidly with decreasing kaon mass. The cross section for kaon production in hot and dense hadronic matter will thus be substantially enhanced. This effect will be studied in the present paper.

This paper is organized as follows. In Sec. II, we discuss how hadron masses are modified in hot, dense nuclear matter and introduce a parametrization for this dependence. In Sec. III, models are introduced for kaon production from hadronic processes in free space. The effect of the medium-modified hadron masses on these processes is given in Sec. IV. The production of kaon from nucleus-nucleus collisions at the AGS energies is studied in Sec. V. In Sec. VI, we discuss necessary improvements which should be made in the future. Finally, the summary is given in Sec. VII.

\section{HADRON MASSES}

\section{A. Nonstrange hadrons}

It is well known that the effective mass of a nucleon in nuclear matter is less than its mass in the free space. For mesons such as $\sigma, \omega$, and $\rho$, Brown has argued that they can be considered as correlated pionic states and their masses are also density dependent ${ }^{26}$ because the pion dispersion relation in nuclear medium becomes softer as a result of the delta-hole polarization of the nuclear matter. ${ }^{27}$ Based on chiral model for the meson-nucleon interaction, one expects that the restoration of chiral symmetry at high density would also lead to the reduction of hadron masses with increasing nuclear density. ${ }^{28}$ The ratio of the hadron mass $m^{*}$ in hadronic matter of baryon density $\rho_{B}$ to its value in the free space $m$ has been parametrized as

$$
m^{*} / m \approx 1-(\lambda / 2)\left(\rho_{B} / \rho_{0}\right)
$$

by Brown. With $\lambda \approx 0.2-0.4$, such density-dependent effective hadron masses can consistently explain recent experimental results on the scattering of kaon, ${ }^{29}$ proton, ${ }^{30}$ and electron ${ }^{31}$ off the nucleus. In normal nuclei, the hadron masses are only reduced by about 10-20\%. Much larger effects will be expected in the hot, dense matter created in high-energy heavy-ion collisions.
As the chiral symmetry is also restored when the temperature of the system is high, hadron masses will decrease with increasing temperature as well. ${ }^{32} \mathrm{~A}$ fundamental approach to the density and temperature dependence of hadron masses requires the solution of the quantum chromodynamics (QCD). Although lattice calculations have not reached the stage that reliable results can be obtained, the QCD sum-rule method ${ }^{33}$ has confirmed the results of the chiral model that hadron masses decrease with increasing density and temperature and give indications that they may vanish when the chiral symmetry is restored at the critical density and temperature. The temperature dependence of the hadron masses obtained by the QCD sum rules can be approximately parametrized by $\left[1-\left(T / T_{c}\right)^{2}\right]^{n}$ with $n=\frac{1}{6}$, where $T_{c}$ is the critical temperature at which the hadronic matter is expected to transform into the quark-gluon plasma. In the chiral models, ${ }^{28}$ the exponent $n$ has a value about $\frac{1}{2}$. For our exploratory study, we shall take $n=\frac{1}{3}$. Calculations with the QCD sum rules ${ }^{34}$ show that the $\rho$-meson changes much more rapidly with density than temperature. To within an accuracy of $\sim 20 \%$, the $\rho$-meson mass scales with density as the nucleon effective mass, namely,

$$
\frac{m_{\rho}^{*}}{m_{\rho}} \approx \frac{m_{N}^{*}}{m_{N}},
$$

indicating that we should choose $\lambda \sim 0.4$ in order to have $m_{N}^{*} / m_{N}=0.8$ at nuclear matter density. Taking both the density and temperature dependence into account simply by multiplying the effects, we have

$$
\frac{m^{*}}{m} \approx\left[1-\left[\frac{T}{T_{c}}\right]^{2}\right]^{n}\left[1-\frac{\lambda}{2}\left(\frac{\rho_{B}}{\rho_{0}}\right)\right] \text {. }
$$

\section{B. Pion}

Because of its special nature as a Goldstone boson, the pion is protected by chiral invariance from large changes in mass. At finite nuclear density $\rho_{N}$, the change in $m_{\pi}^{2}$ is $^{35}$

$$
\delta\left(m_{\pi}^{2}\right)=\frac{g_{\pi N N}^{2} \rho_{N}}{m_{N}}\left(\frac{m_{\pi}}{2 M_{N}}\right)^{2},
$$

giving a $\delta m_{\pi}$ of $\sim 5 \mathrm{MeV}$ for $\rho=\rho_{0}$. The change with temperature is given by ${ }^{36} m_{\pi}\left(1+T^{2} / 48 f_{\pi}^{2}+\cdots\right)$ in the low-temperature expansion, where $f_{\pi}$ is the pion decay constant. Since $T_{c}$ is of order $2 f_{\pi}$, the change again is positive but small. We shall thus ignore the change of the pion mass with density and temperature.

\section{Kaon}

The situation with the kaon mass is of particular interest to us. Although the kaon has a large dynamically generated mass, it behaves in many respects as a Goldstone boson. However, the fact that the strange-quark mass is much larger than the up- and down-quark masses makes it possible to give a straightforward treatment of the kaon effective mass $m_{K}^{*}$ as function of density. 
Kaplan and Nelson ${ }^{25}$ pointed out that, because of the large explicit chiral symmetry breaking (which gives rise to the strange-quark mass), the Lagrangian has a term

$$
L_{I}=\frac{m_{K}^{2}}{2}(\bar{K} K)\left[\frac{\bar{N} N}{\rho_{c}}\right]
$$

where $\rho_{c}$ is the critical density for kaon condensation. This term arises almost completely from the explicit chiral symmetry breaking as incorporated in chiral Lagrangian. It reduces the effective mass of the kaon in dense matter. We can conveniently implement these concepts in the $V$-spin formalism as used by Brown, $\mathrm{Ku}$ bodera, and Rho. ${ }^{37,38}$ We consider condensation in the $V_{0}$ direction, rather than the kaon direction, in order to have chemical potential $\mu=0$. The corresponding quasiparticle is $\frac{1}{2} \pi_{0}+(\sqrt{3} / 2) \eta$, which, from the Gell-MannOkubo mass formula

$$
\frac{1}{4} m_{\pi_{0}}^{2}+\frac{3}{4} m_{\eta}^{2}=m_{K}^{2},
$$

has the same mass as the kaon.

Since the Kaplan-Nelson (KN) strangeness condensation is driven by the $\mathrm{KN}$ sigma term

$$
\Sigma^{\mathrm{KN}}=\frac{1}{2}\left(m_{u}+m_{s}\right)\langle N|\bar{u} u+\overline{s s}| N\rangle,
$$

which explicitly breaks chiral symmetry, the Hamiltonian is given by

$$
H=\rho_{B} \Sigma^{\mathrm{KN}} \cos \theta+\frac{f_{K}^{2}}{2} m_{K}^{2} \sin ^{2} \theta,
$$

with $\theta$ the angle between the scalar axis and the $V_{0}$ direction. Here we have taken the chemical potential to be zero. To consider fluctuation, rather than condensate, kaons, we take $\theta$ to be small; then the $\theta$-dependent part of the Hamiltonian becomes

$$
\begin{aligned}
H(\theta) & =\frac{1}{2} f_{K}^{2} \theta^{2}\left[m_{k}^{2}-\frac{\rho_{B} \Sigma^{\mathrm{KN}}}{f_{K}^{2}}\right] \\
& =\frac{1}{2} f_{K}^{2} \theta^{2} m_{K}^{2}\left[1-\frac{\rho_{B}}{\rho_{c}}\right) .
\end{aligned}
$$

From this, we obtain the kaon effective mass

$$
m_{K}^{*}=m_{K}\left(1-\frac{\rho_{B}}{\rho_{c}}\right)^{1 / 2},
$$

where

$$
\rho_{c}=f_{K}^{2} m_{K}^{2} / \Sigma^{\mathrm{KN}} .
$$

The $m_{K}^{*}$ is smaller than $m_{K}$ because fluctuations in the $\theta$ direction are softened by the decrease in the (repulsive) contribution of $\Sigma^{\mathrm{KN}}$ with increase in $\theta$. Although the bare quark masses may be generated by effects at the $\sim 100 \mathrm{GeV}$ electroweak scale, they, nonetheless, can be numerically compensated for by the kaon rotation at a scale slightly smaller than that of chiral restoration, $\sim 1$ $\mathrm{GeV}$. (Note that the pion mass is "rotated out" at a density $\rho \sim 2.2 \rho_{0}$ by a similar mechanism ${ }^{38}$ but at higher densities the energy of the system is lowered more by ro- tation in the kaon or $V_{0}$ direction.) In the above, one could let $m_{K}^{2} \rightarrow m_{K}^{2}+k_{K}^{2}$ to include kaon kinetic energy.

The important remaining question concerns the size of $\rho_{c}$. Fortunately, we know the size of the $\pi N$ sigma term

$$
\Sigma^{\pi N}=\frac{1}{2}\left(m_{u}+m_{d}\right)\langle N|\bar{u} u+\bar{d} d| N\rangle \approx 53 \mathrm{MeV}
$$

from which we can scale by changing down quark to strange quark in order to obtain $\Sigma^{\mathrm{KN}}$. There is, however, considerable uncertainty in the strangeness content of the nucleon, but we can obtain lower and upper limits on $\rho_{c}$ by taking

$$
\langle N|\bar{s} s| N\rangle=\langle N|\bar{u} u| N\rangle
$$

and by taking $\langle N|\overline{s s}| N\rangle=0$. With $m_{s}=25\left(m_{u}+m_{d}\right) /$ 2 , we can evaluate $\rho_{c}$ from Eq. (10) to find

$$
3 \rho_{0}<\rho_{c}<5 \rho_{0} .
$$

In the above, we have used $f_{K} \approx f_{\pi} \sim 93 \mathrm{MeV}$, which is appropriate because we work only to lowest order in chiral perturbation theory. Although $f_{K}$ becomes somewhat larger than $f_{\pi}$ in first order, both $f_{\pi}$ and $f_{K}$ decrease with density, and the $\bar{s} s$ content of the nucleon will increase. ${ }^{39}$ This would lead to even lower critical density for kaon condensate.

The important point of the preceding development is that densities do not have to be high enough for kaon condensation in order for the kaon effective mass to be substantially lowered. (This was already clear in Ref. 37.) This lowering does not require a large $\bar{s} s$ content in the nucleon, although the effect is increased by a large strangeness content.

For the temperature dependence of the kaon mass, we shall still use the factor $\left[1-\left(T / T_{c}\right)^{2}\right]^{n}$ as for nonstrange hadrons.

\section{Other strange hadrons}

For the mass of other strange hadrons, a substantial part of which comes from explicit chiral symmetry breaking which gives a large current quark mass $m_{s}$. Therefore, we apply the density and temperature factors only to the dynamically generated masses. Their masses are thus parametrized by

$m_{Y}^{*} \approx\left(m_{Y}-m_{s}\right)\left[1-\left[\frac{T}{T_{c}}\right]^{2}\right]^{n}\left[1-\frac{\lambda}{2}\left(\frac{\rho_{B}}{\rho_{0}}\right)\right]+m_{s}$.

We would like to emphasize that the parametrizations introduced in Eqs. (2) and (13) are meant to demonstrate the effect of hot, dense nuclear matter on hadron masses. The detailed form may be different when improved studied are carried out.

Because of such density- and temperature-dependent hadron masses, heavier hadrons will become increasingly important at high density and temperature. Also, a much smoother transition from the hadronic matter to the quark-gluon plasma is expected and this is consistent with results from the lattice gauge calculations. Requir- 
ing the equality of pressures on hadron and quark-gluon plasma sides then gives $T_{c} \approx 195 \mathrm{MeV}$, consistent with chiral perturbation theory. ${ }^{40}$

\section{KAON PRODUCTION IN THE FREE SPACE}

As discussed in the Introduction, kaons can be produced from the three elementary processes: (a) $M M$ $\rightarrow K \bar{K}$, (b) $M B \rightarrow Y K$, and (c) $B B \rightarrow B Y K$. In the following, we discuss how these processes in the free space can be modeled.

\section{A. $M M \rightarrow K \bar{K}$}

Kaons can be produced from the annihilation of mesons. Because of the high abundance of pions and the considered, although if the mass of the rho meson decreases, the mass of the $A_{1}$, its chiral partner, would also be expected to be so. Kaon production from their annihilations is mainly through the exchange diagrams shown in Fig. 1. In general, the isospin-averaged cross section can be expressed as

$$
\bar{\sigma}=\frac{1}{32 \pi} \frac{p^{\prime}}{s p} \int_{-1}^{1} d x M(s, x)
$$

where $s$ is the square of the total center-of-mass energy; $p$ and $p^{\prime}$ are, respectively, the three-momenta of the meson and kaon in the center-of-mass frame, and $M(s, x)$ is the isospin-averaged squared invariant amplitude. In the following, we consider the three Feynmann diagrams in Fig. 1 separately. large degeneracy of the rho meson, only these mesons are

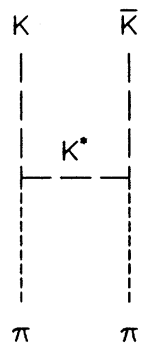

(a)

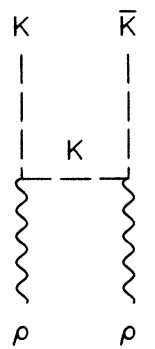

(b)

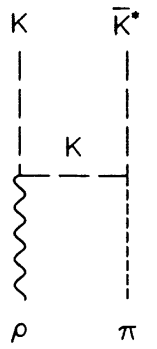

(c)
FIG. 1. Feynman diagrams for $M M \rightarrow K \bar{K}$.

$$
\text { 1. } \pi \pi \rightarrow K \bar{K}
$$

Two pions can annihilate into $K \bar{K}$ through the exchange of a $K^{*}$ as shown in Fig. 1(a). The $K^{*} K \pi$ interaction can be written as

$$
L_{K * K \pi}=g_{K{ }_{K}{ }^{*}} K^{* \mu} \tau\left[K\left(\partial_{\mu} \pi\right)-\left(\partial_{\mu} K\right) \pi\right] .
$$

Carrying out the standard Feynman diagram calculation and taking into account the isospin average, we obtain

$$
\begin{gathered}
M(s, x)=\frac{1}{9}\left[18\left|T\left(q_{+}^{2}\right)\right|^{2}+18\left|T\left(q_{-}^{2}\right)\right|^{2}\right. \\
\left.-12 T_{\text {int }}\left(q_{+}^{2}, q_{-}^{2}\right)\right],
\end{gathered}
$$

where

$$
\begin{aligned}
& T\left(q_{ \pm}^{2}\right)=-g_{K^{*} K \pi}^{2}\left(\frac{s+2 p^{2}+2 p^{\prime 2}+q_{ \pm}^{2}+\left(m_{K}^{2}-m_{\pi}^{2}\right)^{2} / m_{K}^{2}}{q_{ \pm}^{2}-\left(m_{K^{*}}+i \Gamma_{K^{*}} / 2\right)^{2}}\right), \\
& T_{\mathrm{int}}\left(q_{+}^{2}, q_{-}^{2}\right)=\operatorname{Re}\left[T^{*}\left(q_{+}^{2}\right) T\left(q_{-}^{2}\right)\right]
\end{aligned}
$$

with

$$
q_{ \pm}^{2}=m_{\pi}^{2}+m_{K}^{2}-2\left(m_{\pi}^{2}+p^{2}\right)^{1 / 2}\left(m_{K}^{2}+p^{\prime 2}\right)^{1 / 2} \pm 2 p p^{\prime} x
$$

the mass of $K^{*}$, we expect that its contribution is more important. Also, the $\rho K^{*} K$ vertex is more complex than the $\rho K K$ vertex. For simplicity, we shall evaluate only the kaon exchange diagram. The $\rho K K$ interaction is written as

$$
L_{\rho K K}=g_{\rho K K}\left[K \tau\left(\partial_{\mu} K\right)-\left(\partial^{\mu} K\right) \tau K\right] \rho^{\mu} .
$$
tial isospin of the two pions and the three terms correspond to contributions from the direct diagram, the exchange diagram, and their interference.

$$
\text { 2. } \rho \rho \rightarrow K \bar{K}
$$

Two $\rho$ mesons can also annihilate into $K \bar{K}$ by the exchange of either a kaon as shown in Fig. 1(b) or a $K^{*}$. Since the kaon mass is almost a factor of 2 smaller than
The isospin average can be carried out as in the case of $\pi \pi$ annihilation. We then have

$$
\begin{gathered}
M(s, x)=\frac{1}{81}\left[18\left|T\left(q_{+}^{2}\right)\right|^{2}+18\left|T\left(q_{-}^{2}\right)\right|^{2}\right. \\
\left.-12 T_{\text {int }}\left(q_{+}^{2}, q_{-}^{2}\right)\right],
\end{gathered}
$$

where

$$
\begin{aligned}
& T\left(q_{ \pm}^{2}\right)=g_{\rho K K}^{2}\left[\frac{m_{\rho}^{2}-2 m_{K}^{2}-2 q_{ \pm}^{2}+\left(m_{K}^{2}-q_{ \pm}^{2}\right)^{2} / m_{\rho}^{2}}{q_{ \pm}^{2}-m_{k}^{2}}\right], \\
& T_{\text {int }}\left(q_{+}^{2}, q_{-}^{2}\right)=g_{\rho K K}^{4} \frac{\left[-\left(s+m_{\rho}^{2}-4 m_{K}^{2}\right)+\left(q_{+}^{2}-m_{K}^{2}\right)\left(q_{-}^{2}-m_{K}^{2}\right) / m_{\rho}^{2}\right]^{2}}{\left(q_{+}^{2}-m_{K}^{2}\right)\left(q_{-}^{2}-m_{K}^{2}\right)} .
\end{aligned}
$$


In the above, $q_{ \pm}$is similarly defined as Eq. (17) except $m_{\pi}$ is replaced by $m_{\rho}$. The term $T_{\text {int }}$ comes from the interference contribution. We note that

$$
T_{\text {int }}\left(q_{+}^{2}, q_{-}^{2}\right) \neq T\left(q_{+}^{2}\right) T\left(q_{-}^{2}\right)
$$

because $T_{\text {int }}\left(q_{+}^{2}, q^{2}\right)$ is obtained by summing over the $\rho$ meson polarization after taking the product of the direct and exchange amplitudes. The factor $\frac{1}{81}$ in Eq. (18) is again due to the average over the initial isospin and polarization of the two rho mesons.

$$
\text { 3. } \pi \rho \rightarrow K \bar{K}^{*} \text { and } \pi \rho \rightarrow K^{*} \bar{K}
$$

Furthermore, a pion and a $\rho$ meson can annihilate into $K \bar{K}^{*}$ as shown in Fig. 1(c) or they can annihilate into $K \bar{K}$ via the exchange of the $K^{*}$. Although the phase space for the latter is more favorable, it has a smaller matrix element because of the larger $K^{*}$ mass in the propagator. For simplicity, we neglect its contribution. The diagram in Fig. 1(c) also implies the annihilation into $K^{*} \bar{K}$. The isospin-averaged squared invariant amplitude is given by

$$
\begin{aligned}
M(s, x)= & \frac{2}{3} g_{\rho K K}^{2} g_{K^{*} K \pi}^{2} \frac{1}{\left(q^{2}-m_{K}^{2}\right)^{2}} \\
& \times\left[m_{K^{*}}^{2}-2 m_{\pi}^{2}-2 q^{2}+\left(m_{\pi}^{2}-q^{2}\right)^{2} / m_{K^{*}}^{2}\right] \\
& \times\left[m_{\rho}^{2}-2 m_{K}^{2}-2 q^{2}+\left(m_{K}^{2}-q^{2}\right) / m_{\rho}^{2}\right], \\
q^{2}=m_{\pi}^{2} & +m_{K^{*}}^{2}-2\left(m_{\pi}^{2}+p^{2}\right)^{1 / 2}\left(m_{K^{*}}^{2}+p^{\prime 2}\right)^{1 / 2}+2 p p^{\prime} x
\end{aligned}
$$

Since there is only the direct diagram for this process, a factor of 18, as in both Eqs. (15) and (18), is obtained from the isospin sum. The average over the initial spin and isospin gives a factor $\frac{1}{27}$. The factor $\frac{2}{3}$ in Eq. (20) then follows by combining the two.

Because of the finite size of hadrons, form factors need to be introduced in the vertices. Following Lohse et al. ${ }^{22}$ we use the monopole form

$$
F_{\alpha}\left(\mathbf{q}^{2}\right)=\frac{\Lambda^{2}-m_{\alpha}^{2}}{\Lambda^{2}+\mathbf{q}^{2}},
$$

where $m_{\alpha}$ is the mass of the exchanged particle and $\Lambda$ is the cutoff parameter.

To evaluate the kaon production cross section, we need to know the values of the coupling constants and cutoff parameters. The coupling constant $g_{K}{ }_{K \pi}$ can be determined from the measured width of $K^{*}$ via

$$
\Gamma_{K} *=\frac{g_{K}^{2} K_{\pi}}{2 \pi} \frac{k^{3}}{m_{K^{*}}^{2}},
$$

with $m_{K^{*}}$ being the mass of $K^{*}$ and $k$ being the momentum of the kaon in its rest frame. From $\Gamma_{K^{*}} \sim 51 \mathrm{MeV}$, a value of $g_{K}^{2}{ }_{K \pi} /(4 \pi) \sim 0.849$ is obtained.

From SU(3), the coupling constant $g_{\rho K K}$ is half of the $\rho \pi \pi$ coupling constant. ${ }^{22}$ The latter can be determined from the width of the $\rho$ meson

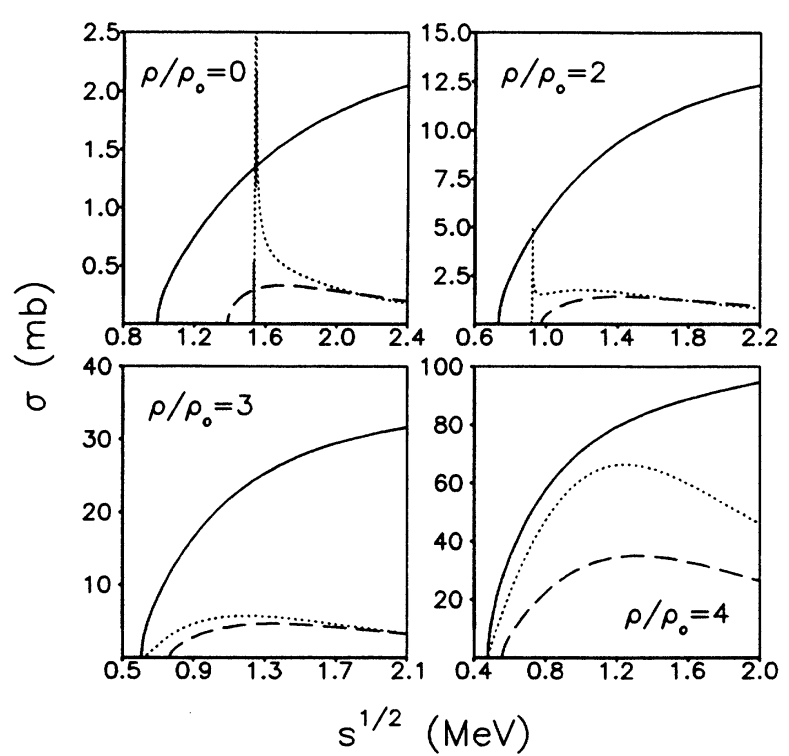

FIG. 2. Energy dependence of the cross sections for $\pi \pi \rightarrow K \bar{K}$ (solid curve), $\rho \rho \rightarrow K \bar{K}$ (dotted curve), and $\pi \rho \rightarrow K \bar{K}$ (dashed curve) at different nuclear densities.

$$
\Gamma_{\rho}=\frac{g_{\rho \pi \pi}^{2}}{6 \pi} \frac{k^{3}}{m_{\rho}^{2}},
$$

where $m_{\rho}$ is the rho-meson mass and $k$ is the momentum of the pion in its rest frame. Taking $\Gamma_{\rho} \sim 153 \mathrm{MeV}$, one obtaines $g_{\rho \pi \pi}^{2} /(4 \pi) \sim 2.94$ which gives $g_{\rho K K}^{2} /$ $(4 \pi) \sim 0.735$. As in Ref. 22, the value of $g_{\rho K K}$ is similar to that of $g_{K^{*}{ }_{K} \pi}$. But our values for the coupling constants are larger than the value 0.525 of Ref. 18 in which more detailed coupled-channel studies have been carried out. The cutoff parameter at the vertex $K^{*} K \pi$ is determined to be $3 \mathrm{GeV}$ in Ref. 22 from fitting the empirical data. The kaon production cross section using Eq. (15) and the coupling constant and cutoff parameter of Ref. 22 can be reproduced with our coupling constant and a cutoff parameter of $1.8 \mathrm{GeV}$. In the following, we shall use our coupling constants determined from the $K^{*}$ and $\rho$ widths and the cutoff parameter of $1.8 \mathrm{GeV}$ at both the $K^{*} K \pi$ and $\rho K K$ vertices.

In the upper left panel of Fig. 2, we show the energy dependence of the three meson-meson annihilation cross sections in free space $\left(\rho / \rho_{0}=0\right)$; the solid curve is for $\pi \pi \rightarrow K \bar{K}$, the dashed curve is for $\pi \rho \rightarrow K \bar{K}$, and the dotted curve is for $\rho \rho \rightarrow K \bar{K}$. In the energy region of interest, near the threshold, all calculated cross sections are less than $1 \mathrm{mb}$. Since the rho-meson mass is larger than the kaon mass, the $\rho \rho$ cross section has a singularity at the threshold. The cross section for $\pi \pi \rightarrow K \bar{K}$ is most important but it is still quite small in the relevant range of energies. In the above, we have neglected the $s$-channel process in which the isoscalar resonance $f_{0}(975)$ is formed from pion-pion annihilation and then decays into the kaon-antikaon pair as this cross section is not important after averaging over the pion isospin. Also, its contribution becomes negligible with increasing energy because of the narrow width (33 MeV) of $f_{0}$. 


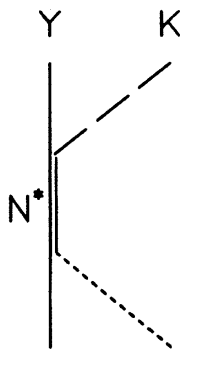

N

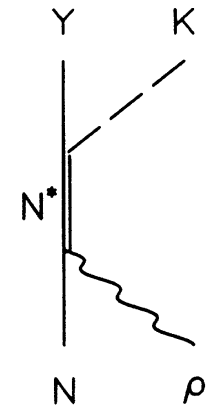

(b)
FIG. 3. Feynman diagram for $M B \rightarrow Y K$.

\section{B. $M B \rightarrow Y K$}

Again, we shall consider only kaon production from the pion and rho meson as shown in Fig. 3. For $\pi N \rightarrow \Lambda K$, we include intermediate states such as $N_{1}^{*}(1650), N_{2}^{*}(1710)$, and $N_{3}^{*}(1720)$ as they have appreciable probability of decaying into $\Lambda K$. Neglecting the interference, the isospin-averaged cross section can be expressed as

$\bar{\sigma}_{\pi N \rightarrow \Lambda K}=\sum_{i} \frac{\left(2 J_{i}+1\right)}{2} \frac{\pi}{k_{i}^{2}} \frac{B_{i \pi N} B_{i \Lambda K} \Gamma_{i}^{2}}{\left(s^{1 / 2}-m_{i}\right)^{2}+\Gamma_{i}^{2} / 4}$,

with $s^{1 / 2}$ being the center-of-mass energy. The summation is over the three resonances. The mass, width, and spin of the $i$ th resonance are denoted by $m_{i}, \Gamma_{i}$, and $J_{i}$, respectively. The branching fractions of the $i$ th resonance into the $\pi N$ and $\Lambda K$ channels are given by $B_{i \pi N}$ and $B_{i \Lambda K}$. The numerical values for these parameters are taken from the particle properties data booklet and are collected in Table I.

Similarly, the cross section for $\rho N \rightarrow \Lambda K$ can be calculated from Eq. (25) by substituting $B_{i \rho N}$ for $B_{i \pi N}$. Since the mass of $N_{1}^{*}$ is less than the sum of the rho-meson and nucleon masses, we do not include its contribution in the present study. Its contribution can be included by averaging Eq. (25) over the rho-meson mass distribution.

In the upper left panel of Fig. 4, the energy dependence

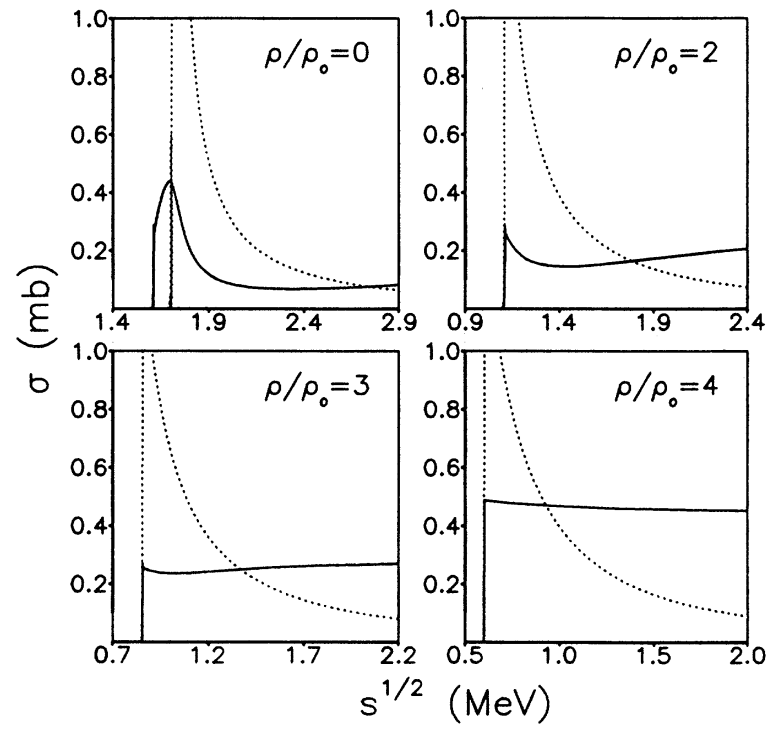

FIG. 4. Energy dependence of the cross sections for $\pi N \rightarrow \Lambda K$ (solid curve) and $\rho N \rightarrow \Lambda K$ (dashed curve) at different nuclear densities.

of the cross sections for $\pi N \rightarrow \Lambda K$ and $\rho N \rightarrow \Lambda K$ in free space are shown by the solid and dashed curves, respectively. Our results agree reasonably with the empirical data $^{12}$ for $\pi N \rightarrow \Lambda K$.

The interaction of pions and rho mesons with other baryons can also produce kaons. Since the three resonances mentioned above have the largest branching factors for decaying into a kaon, we assume that they are involved in kaon production from all meson-baryon interactions. Then Eq. (25) can be used with the center-ofmass energy $s^{1 / 2}$ calculated according to the corresponding baryon masses.

\section{C. $B B \rightarrow B Y K$}

Kaon production from the baryon-baryon interaction can be approximately described by the one-pion-exchange model. For $N N \rightarrow N \Lambda K$, the corresponding Feynman diagram is shown in Fig. 5. The isospin-averaged cross section is then given by ${ }^{5,10}$

$$
\bar{\sigma}_{N N \rightarrow N \Lambda K}=\frac{3 m_{N}^{2}}{2 \pi^{2} p^{2} s} \int_{W_{\min }}^{W_{\max }} d W W^{2} k \int_{q_{-}^{2}}^{q_{+}^{2}} d q^{2} \frac{f_{\pi N N}^{2}}{m_{\pi}^{2}} F^{2}\left(q^{2}\right) \frac{q^{2}}{\left(q^{2}-m_{\pi}^{2}\right)^{2}} \bar{\sigma}_{0}\left(W ; q^{2}\right)
$$

TABLE I. Parameters for baryon resonances. $m, J, L, \Gamma$, and $B$ denote, respectively, the mass, spin, orbital angular momentum, total width, and branching factor.

\begin{tabular}{ccccccc}
\hline \hline$m(\mathrm{MeV})$ & $J$ & $L$ & $\Gamma(\mathrm{MeV})$ & $B_{\pi N}$ & $B_{\rho N}$ & $B_{\Lambda K}$ \\
\hline 1650 & $\frac{1}{2}$ & 0 & 150 & 0.60 & 0.18 & 0.08 \\
1710 & $\frac{1}{2}$ & 1 & 110 & 0.15 & 0.20 & 0.15 \\
1720 & $\frac{3}{2}$ & 1 & 200 & 0.15 & 0.75 & 0.08 \\
\hline \hline
\end{tabular}




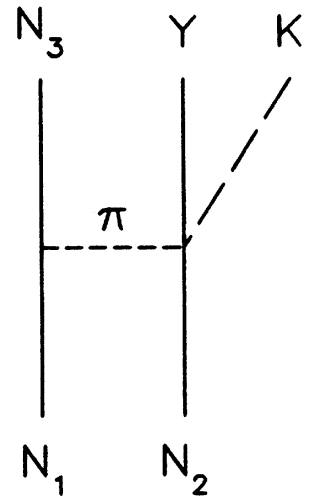

FIG. 5. Feynman diagram for $B B \rightarrow B Y K$.

In the above, the square of the total energy of the initial nucleons in the center-of-mass system is denoted by $s$. With $W$ being the total energy in the center-of-mass system of the pion and $N_{2}$, one has $W_{\min }=m_{K}+m_{\Lambda}$, $W_{\max }=s^{1 / 2}-m_{N}$, and

$$
q_{ \pm}^{2}=2 m_{N}^{2}-2 E E^{\prime} \pm 2 p p^{\prime},
$$

where $p$ and $E=\left(p^{2}+m_{N}^{2}\right)^{1 / 2}$ are the energy and momentum of nucleon $N_{1}$ in the center-of-mass system while $p^{\prime}$ and $E^{\prime}$ are those of nucleon $N_{3}$, respectively. The momentum of the pion in this frame is denoted by $k$. The coupling constant $f_{\pi N N}$ has a value of one and the form factor is taken to be

$$
F=\left(\Lambda^{2}-m_{\pi}^{2}\right) /\left(\Lambda^{2}-q^{2}\right),
$$

with $\Lambda$ being the cutoff parameter. The quantity $\bar{\sigma}_{0}\left(W ; q^{2}\right)$ is the isospin-averaged cross section for the virtual process $\pi N_{2} \rightarrow \Lambda K$ which are approximated by the on-shell cross section and is taken from Sec. III B.

Using a cutoff parameter $\Lambda=1 \mathrm{GeV}$, the calculated kaon production cross section from $N N \rightarrow N \Lambda K$ in free space is shown by the solid curve in the upper left panel of Fig. 6 as a function of the center-of-mass energy. For the energy range we are interested in, the one-pionexchange model gives a reasonable description of the data. ${ }^{10}$

Kaons can also be produced from nucleon-delta and delta-delta interactions. The corresponding cross sections have been estimated in Ref. 10 and it was found that

$$
\bar{\sigma}_{N \Delta \rightarrow N \Lambda K} \approx \frac{3}{4} \bar{\sigma}_{N N \rightarrow N \Lambda K}
$$

and

$$
\bar{\sigma}_{\Delta \Delta \rightarrow N \Lambda K} \approx \frac{1}{2} \bar{\sigma}_{N N \rightarrow N \Lambda K}
$$

at the same center-of-mass energy. For kaon production from the interactions between other baryons, we approximate the cross section by $\bar{\sigma}_{\Delta \Delta \rightarrow N \Lambda K}$.

\section{KAON PRODUCTION IN THE MEDIUM}

The free cross sections shown in the above are modified when the medium-modified hadron masses as given by

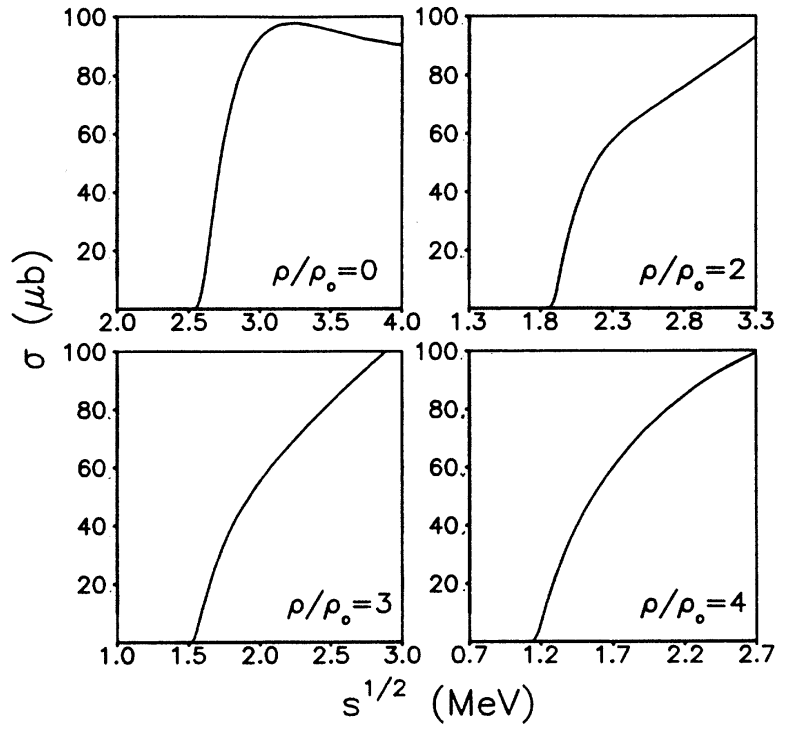

FIG. 6. Energy dependence of the cross sections for $N N \rightarrow N \Lambda K$ at different nuclear densities.

Eqs. (2), (9), and (13) are used. To illustrate this effect, we first consider only the density effect by neglecting the temperature dependence of the hadron masses. In Fig. 2, we show the energy dependence of the kaon production cross section from $M M \rightarrow K \bar{K}$ for different nuclear matter densities. It is seen that all cross sections are substantially enhanced in high-density matter. The density dependence of $M B \rightarrow Y K$ is shown in Fig. 4 while that of $B B \rightarrow B Y K$ is shown in Fig. 6. Their density dependence is, however, not appreciable.

To see the combined effect of density and temperature, we calculate $\left\langle\sigma_{12}^{f} v_{12}\right\rangle$ by averaging the product of the cross section $\sigma_{12 \rightarrow f}$ and the relative velocity $v_{12}$ over particle distributions, assuming that they are in thermal equilibrium, i.e.,

$$
\left\langle\sigma_{12}^{f} v_{12}\right\rangle=\int d^{3} \mathbf{k}_{1} \int d^{3} \mathbf{k}_{2} f_{1}\left(\mathbf{k}_{1}\right) f_{2}\left(\mathbf{k}_{2}\right) \sigma_{12}^{f} v_{12} .
$$

In the above, $f$ 's are the normalized momentum distributions of the particles. Details on the evaluation of Eq. (27) can be found in Ref. 16.

In the following calculations, we use $n=\frac{1}{3}, \lambda=0.3$, $T_{c}=195 \mathrm{MeV}$, and $\rho_{c}=5 \rho_{0}$. The results are shown in Figs. 7-9 for $\left\langle\sigma_{M M}^{K \bar{K}} v_{M M}\right\rangle,\left\langle\sigma_{M B}^{Y K} v_{M B}\right\rangle$, and $\left\langle\sigma_{B B}^{B Y K} v_{B B}\right\rangle$, respectively.

In Fig. 7, the dotted, short-dashed, and long-dashed curves correspond, respectively, to $\pi \pi \rightarrow K \bar{K}, \pi \rho \rightarrow K \bar{K}{ }^{*}$, and $\rho \rho \rightarrow K \bar{K}$. Although the cross section for $\rho \rho \rightarrow K \bar{K}$ has a singularity at the threshold as shown in Fig. 2(a), it leads to finite results when one calculates the thermal average. Also shown in the figure is $\left\langle\sigma_{M M}^{K \bar{K}} v_{M M}\right\rangle$ which is obtained by averaging over the initial hadronic states assuming that they are in equilibrium, i.e.,

$$
\left\langle\sigma_{M M}^{K \bar{K}} v_{M M}\right\rangle=\sum_{i, j} \rho_{i} \rho_{j}\left\langle\sigma_{i j}^{K \bar{K}} v_{i j}\right\rangle /\left[\sum_{i} \rho_{i}\right]^{2},
$$

where the summation is over pion and rho meson. At 

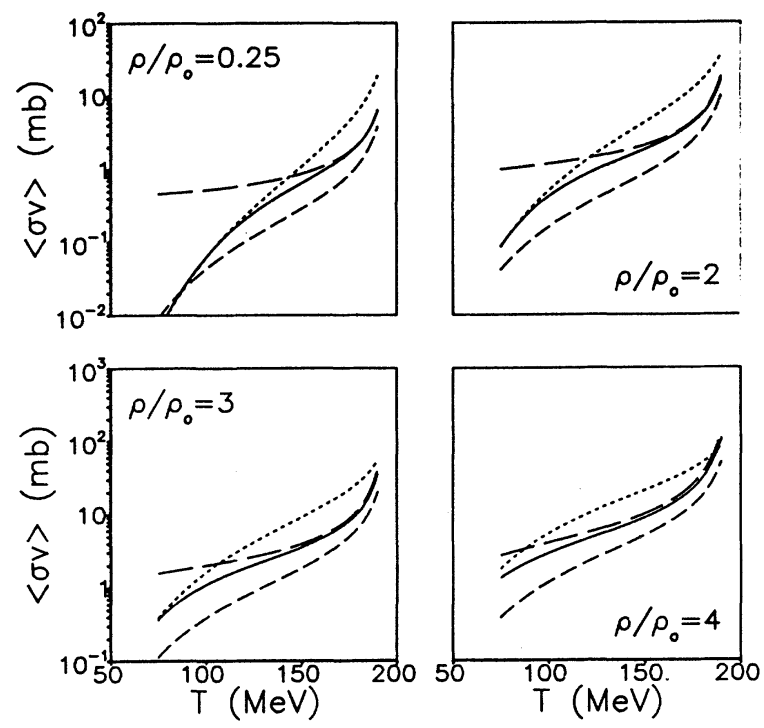

FIG. 7. The density and temperature dependence of $\langle\sigma v\rangle$ for $\pi \pi \rightarrow K \bar{K}$ (dotted curve), $\pi \rho \rightarrow K \bar{K}$ (short-dashed curve), and $\rho \rho \rightarrow K \bar{K}$ (long-dashed curve). The solid curve is from $M M \rightarrow K \bar{K}$ by averaging over the initial meson distributions.

low densities where hadron masses are essentially their bare masses, both $\left\langle\sigma_{\pi \pi}^{K \bar{K}} v\right\rangle$ and $\left\langle\sigma_{\pi \rho}^{K \bar{K}} v\right\rangle$ increase with increasing temperature while $\left\langle\sigma_{\rho \rho}^{K \bar{K}} v\right\rangle$ does not change appreciably. This illustrates the thermal effect on the kaon production cross section. All are enhanced significantly at high densities where the hadron masses decrease substantially.

In Fig. 8, we show by the dotted-and long-dashed curves the results from $\pi N \rightarrow \Lambda K$ and $\rho N \rightarrow \Lambda K$. The solid curve is from $M B \rightarrow \Lambda K$ by averaging over the initial meson and baryon distributions defined by an equa-
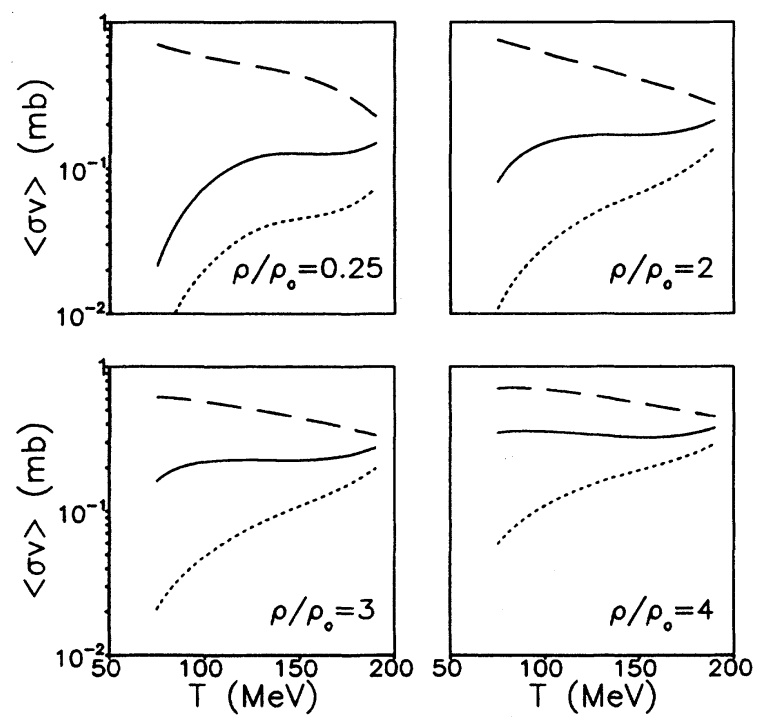

FIG. 8. Same as Fig. 7 for $\pi N \rightarrow \Lambda K$ (dotted curve), $\rho N \rightarrow \Lambda K$ (long-dashed curve), and $M B \rightarrow \Lambda K$ (solid curve).
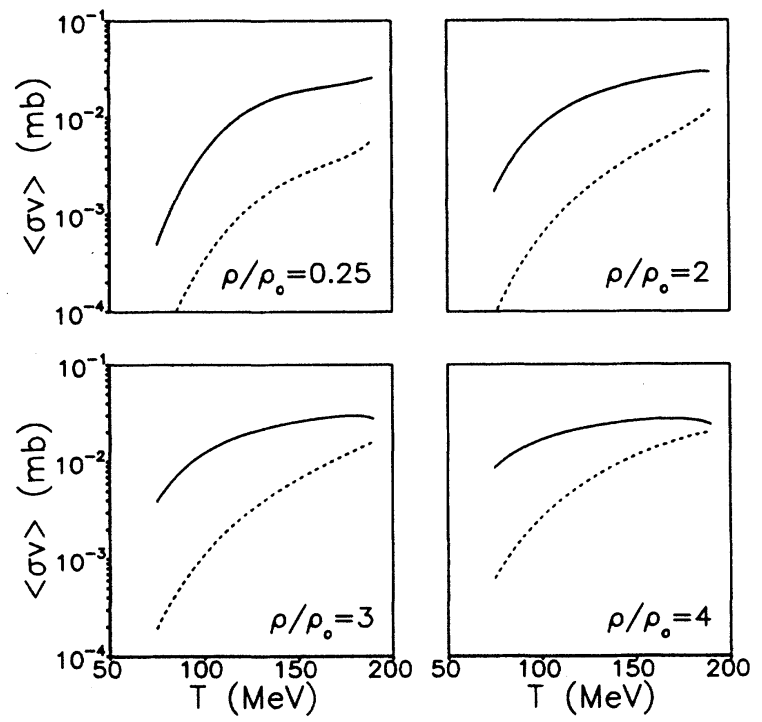

FIG. 9. Same as Fig. 7 for $N N \rightarrow N \Lambda K$ (dotted curve) and $B B \rightarrow B \Lambda K$ (solid curve).

tion similarily to Eq. (28). Although there is some density effect at low temperature, it is not appreciable at high temperature. The effect of decreasing hadron masses on $M B \rightarrow Y K$ is thus not very important compared to its effect on $M M \rightarrow K \bar{K}$.

In Fig. 9, the results for $N N \rightarrow N \Lambda K$ are given by the dotted curve. Averaging over the initial baryon states, we obtain the solid curve for $B B \rightarrow N \Lambda K$. Again, the effect of decreasing hadron masses is not appreciable in this case.

From the above results, we see that $M M \rightarrow K \bar{K}$ is much more important than $M B \rightarrow \Lambda K$ and $B B \rightarrow B Y K$. Also, the process $\rho \rho \rightarrow K \bar{K}$ is very important at high temperature and density.

\section{KAON PRODUCTION FROM NUCLEUS-NUCLEUS COLLISIONS}

\section{A. Hydrochemical model}

To model the collision between two nuclei at high energies, we assume that a fireball is formed with the number of participants determined by the geometry. The initial baryon and energy densities can then be estimated by assuming that the two nuclei are fully stopped. For collisions between identical nuclei at about $15 \mathrm{GeV} /$ nucleon, the initial baryon and energy densities are about $6 \rho_{0}$ and $3 \mathrm{GeV} / \mathrm{fm}^{3}$, respectively. For the collision between $\mathrm{Si}$ and $\mathrm{Au}$ that is currently available at the AGS, it is not clear how to determine these quantities properly. Assuming that the fireball volume is given by the arithmetic mean of two Lorentz-contracted volumes, we obtain that the initial baryon and energy densities are about $4.5 \rho_{0}$ and $1.9 \mathrm{GeV} / \mathrm{fm}^{3}$, respectively. In reality, these numbers could be smaller. The same model has been used previously in Ref. 16.

To describe the fireball expansion, we use the hydro- 
dynamical model of Birö et al. ${ }^{41}$ in which the fireball is assumed in thermal equilibrium and the thermal energy in the fireball is converted into collective flow energy via the relativistic hydrodynamical equations which are further coupled to the rate equations

$$
\begin{aligned}
& \partial_{\mu} T^{\mu 0}=0, \\
& u_{\nu} \partial_{\mu} T^{\mu v}=0, \\
& \partial_{\mu}\left(\rho_{i} u^{\mu}\right)=\Psi_{i},
\end{aligned}
$$

where $T^{\mu v}$ is the energy-momentum tensor and is given by

$$
T^{\mu v}=(e+p) u^{\mu} u^{v}-p g^{\mu v} .
$$

In the above, $e$ and $p$ are, respectively, the energy density and pressure, $g^{\mu \nu}$ is the standard metric tensor, $u^{\mu}$ is the four-velocity defined by $u^{\mu}=\gamma(1, \mathbf{u})$ with $\mathbf{u}$ the local velocity and $\gamma=1 /\left(1-u^{2}\right)^{1 / 2}$ the associated Lorentz factor, and $\Psi_{i}$ is the source term for particle density $\rho_{i}$. We assume that the fireball has a uniform density and the hydrodynamical equations can then be simplified by taking the spatial average. In calculating the energy and pressure densities, we use the noninteracting particle model but with the hadron masses given by Eqs. (2), (9), and (13).

In Ref. 16, chemical equilibrium is not assumed. Due to the enhanced interactions among nonstrange hadrons as a result of the reduced masses in hot, dense nuclear matter, it is reasonable to assume that they are also in chemical equilibrium. Because of the stronger interactions for the antikaon and strange baryons such as $\Lambda(1115), \Sigma(1193)$, and $\Sigma(1385)$, we assume that they are also in chemical equilibrium. But kaons are not in chemical equilibrium and their abundance is determined by the rate equation

$$
\begin{aligned}
\Psi_{K}= & \frac{1}{2}\left\langle\sigma_{M M}^{K \bar{K}} v_{M M}\right\rangle \rho_{M}^{2}-\left\langle\sigma_{K K}^{M M} v_{K \bar{K}}\right\rangle \rho_{K} \rho_{\bar{K}} \\
& +\left\langle\sigma_{M B}^{Y K} v_{M B}\right\rangle \rho_{M} \rho_{B}-\left\langle\sigma_{Y K}^{M B} v_{Y K}\right\rangle \rho_{Y} \rho_{K} \\
& +\frac{1}{2}\left\langle\sigma_{B B}^{B Y K} v_{B B}\right\rangle \rho_{B}^{2},
\end{aligned}
$$

which can be written as

$$
\begin{aligned}
\Psi_{K}= & \frac{1}{2}\left\langle\sigma_{M M}^{K \bar{K}} v_{M M}\right\rangle \rho_{M}^{2}\left[1-\frac{\rho_{M}^{(0)^{2}} \rho_{K} \rho_{\bar{K}}}{\rho_{M}^{2} \rho_{K}^{(0)} \rho_{\bar{K}}^{(0)}}\right] \\
& +\left\langle\sigma_{M B}^{Y K} v_{M B}\right\rangle \rho_{M} \rho_{B}\left[1-\frac{\rho_{M}^{(0)} \rho_{B}^{(0)} \rho_{Y} \rho_{K}}{\rho_{M} \rho_{B} \rho_{Y}^{(0)} \rho_{K}^{(0)}}\right) \\
& +\frac{1}{2}\left\langle\sigma_{B B}^{B Y K} v_{B B}\right\rangle \rho_{B}^{2} .
\end{aligned}
$$

In the above, the density $\rho^{(0)}$ denotes the value at equilibrium.

\section{B. Results for $\mathrm{Si}+\mathrm{Au}$ at $14.6 \mathrm{GeV} /$ nucleon}

Using an initial baryon and energy densities of $4 \rho_{0}$ and $1.4 \mathrm{GeV} / \mathrm{fm}^{3}$, respectively, that are slightly lower than those estimated above, we have solved the hydrochemical equations and the results are shown in Figs. 10-14. Fig-

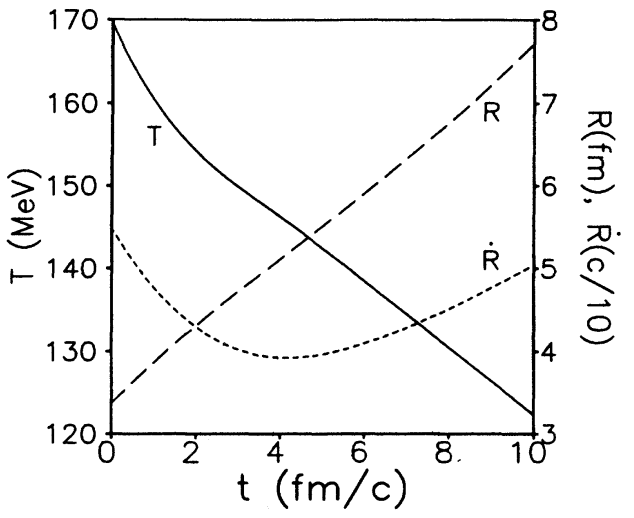

FIG. 10. The time evolution of temperature $T$, radius $R$, and the collective flow velocity $\dot{R}$ of the fireball.

ure 10 shows the time evolution of temperature $T$, radius $R$, and the collective flow velocity $\dot{R}$ of the fireball. The initial temperature of the fireball is about $170 \mathrm{MeV}$ and is below the critical temperature. Because the initial internal energy is lower than the available total center-of-mass energy, the flow energy is nonzero and leads to an initial flow velocity of about $0.55 \mathrm{c}$. As the system expands and cools, hadron masses have to increase. Some of the energies required for the mass increase come from the flow energy as seen by the initial decrease of the flow velocity. When the hadron masses are close to their bare masses as shown in Fig. 11, the flow velocity starts to increase again due to the conversion of the thermal energy to the flow energy.

The time when a particle freezes out from the fireball depends on its transport mean free path and is thus different for differnt kinds of particles. In the present study, we assume for simplicity that all particles freeze out at the same time when the fireball density is about $\frac{1}{3}$ the normal nuclear matter density. This occurs at about $10 \mathrm{fm} / c$ after the expansion when the temperature is about $125 \mathrm{MeV}$ and the collective flow velocity is about $0.5 c$. These values are very similar to those of Lee et al. ${ }^{42}$ who have more carefully treated the freeze out

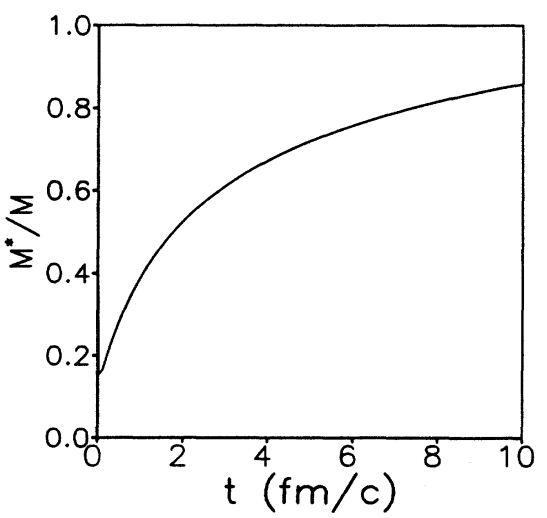

FIG. 11. The time evolution of the hadron masses given by Eq. (2). 


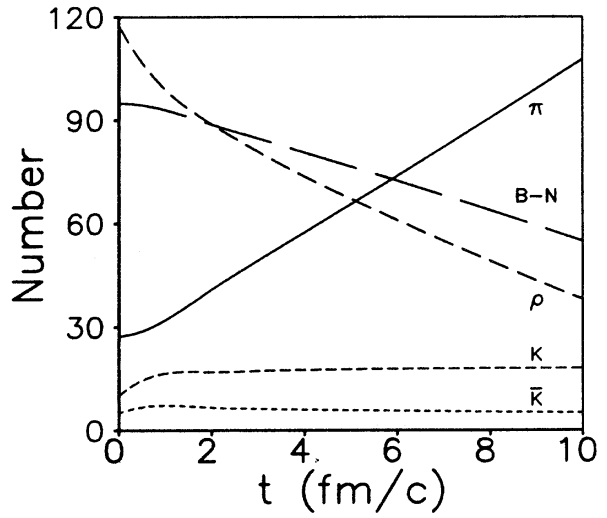

FIG. 12. The time evolution of the particle abundance.

conditions in the hydrodynamical model.

Figure 12 gives the time evolution of the abundance of baryon resonances $(B-N)$, pion $(\pi)$, rho meson $(\rho)$, kaon $(K)$, and antikaon $(\bar{K})$. The number of strange baryons is then given by the difference of the kaon and antikaon numbers. To obtain the nucleon number, we simply subtract the number of baryon resonances and strange baryons from the total number of baryons in the fireball which is about 102. We see that, initially, there are more rho mesons and higher baryon resonances than pions and nucleons because of the reduction of hadron masses at high density and temperature. The initial number of kaons and antikaons is taken to be about ten and four, respectively, to simulate their production from the nonequilibrium stage of the collision. This would give the ratios $K^{+} / \pi \sim 10 \%$ and $K^{-} / \pi^{-} \sim 4 \%$ if chemical reactions are neglected in the expansion. These ratios are similar to those from the proton-nucleus interaction at the same energy. Most kaons are produced during the

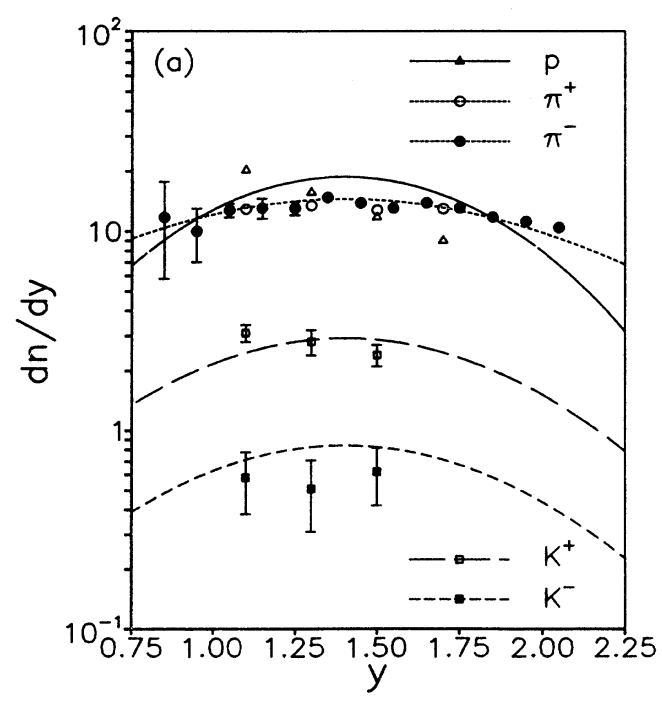

FIG. 13. The rapidity distribution of particles. Curves are from the theoretical calculations while data are from Ref. 14. first $1 \mathrm{fm} / c$ when the density and temperature of the fireball are high and are mainly from the process $\rho \rho \rightarrow K \bar{K}$ because of the initial large rho-meson density.

To obtain the momentum distribution of particles, we need to fold their thermal distribution at freeze out with the collective flow velocity. ${ }^{43}$ In Figs. 13 and 14, we show the rapidity and transverse kinetic-energy distributions of particles and compare them to the measured data. Pions from the decay of baryons are not included as they have transverse momenta largely below the experimental cutoffs. For the rapidity distribution, we see that the pion distribution agrees very well with the data but the peaking of the proton distribution at the central rapidity is at variance with the measured proton distribution which increases instead with decreasing rapidity. The latter is due to our neglect of protons from the target spectator. Both kaon and antikaon rapidity distributions agree fairly with the data. The theoretical kaon distribution again centers at the central rapidity while the measured one centers at lower rapidity. This can be explained if we allow some of the initial kaons to be produced from the interaction of pions with the target spectator nucleons. As to the transverse kinetic-energy distribution, all particles have essentially exponential distributions and agree with the data except for protons, which has a flatter slope than that of the data. The agreement will be improved if we properly include the cool protons from decays of higher baryon resonances at freeze out. ${ }^{44}$

In normal nuclear matter, kaons have a longer mean free path in nuclear matter than other hadrons. But the process $K \pi \leftrightarrow K^{*}$ is important in hot and dense hadronic matter which consists of more pions than nucleons. The isospin-averaged cross section ${ }^{45}$ for this process is about $60 \mathrm{mb}$ and it is thus reasonable to assume that kaons also freeze out in the later stage of the expansion when the density is low.

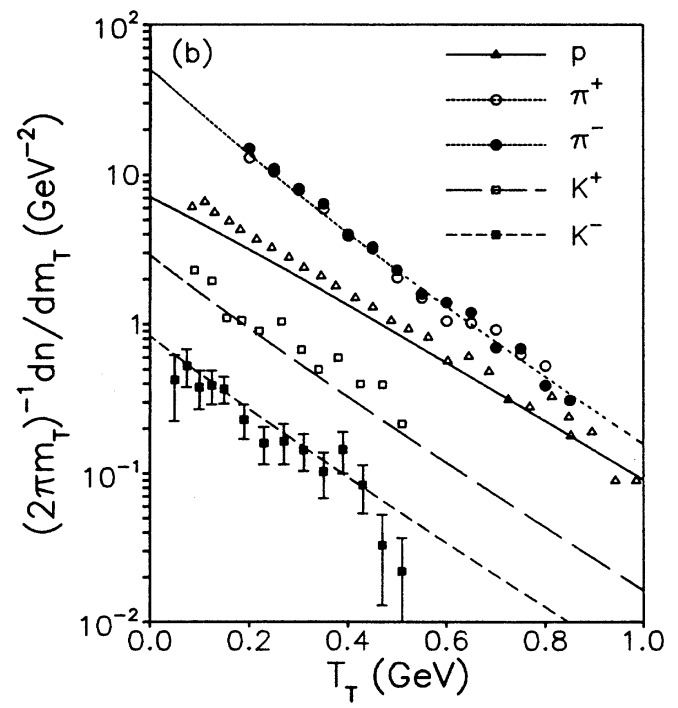

FIG. 14. Same as Fig. 13 for the transverse kinetic-energy distribution of particles. 


\section{NECESSARY IMPROVEMENTS}

Although the preceding development brings out our main point that decreased meson masses, especially decreased kaon masses, facilitate kaon production at high densities, our development is not thermodynamically consistent because we have simply changed the masses in the noninteracting particle model without introducing corrections to the energy and pressure of the system from the particle interactions.

In the case of the nucleons, these effects can be included, as in the Walecka model, ${ }^{46}$ using the relativistic Vlasov-Uehling-Uhlenbeck formalism. ${ }^{47}$ The nucleon effective mass is then connected to the scalar field while its energy is shifted by the vector field. Although there is considerable cancellation between effects from scalar and vector fields, at high densities some of the energy is in these fields so that the initial temperature of the system is lower than that of the free-particle system. But the pressure of the interacting system is also lower compared to the free-particle pressure, leading to a slower initial expansion of the system. Although the kaon yield is reduced by lower temperatures, it is increased by the slower expansion. We therefore expect to see an enhanced kaon production in more realistic studies as well.

To include other particles, one can use the model developed recently by Lynn, Nelson, and Tetradis. ${ }^{48}$ They extend the Walecka model to include $\mathrm{SU}(3)_{L} \times \mathrm{SU}(3)_{R}$ chiral symmetry by coupling the scalar and vector fields to all baryons. The interaction of the pseudo-Goldstone bosons, resulting from the spontaneous breaking of the chiral symmetry, is taken into account by coupling them to the baryons using a nonlinear sigma model. The pseudo-Goldstone bosons are also allowed to interact with the scalar and vector fields. With such a model, it will be possible to derive the density and temperature dependence off hadron effective masses and to treat the thermodynamics of the system in a consistent framework.

\section{SUMMARY}

The creation of a hot, dense nuclear matter in the initial stage of heavy-ion collisions provides us with the unique opportunity to study how the properties of hadrons and their interactions are modified in such a medium. The recent experimental results on kaon production at the AGS suggests that the pion-pion annihilation plays an important role. From chiral models and QCD sum rules, hadron masses are expected to decrease when the density and temperature of the matter are high. With such density- and temperature-dependent hadron masses, the cross section for the process $M M \rightarrow K \bar{K}$ is seen to increase significantly in hot, dense nuclear matter. In particular, the contribution from $\rho \rho \rightarrow K \bar{K}$ becomes most important not only because of the enhanced cross section but also its large abundance as a result of the decreasing hadron masses. The effect of decreasing hadron masses on kaon production from other processes such as $M B \rightarrow \Lambda K$ and $B B \rightarrow B \Lambda K$ is less appreciable. Using the hydrochemical model, we have calculated the kaon yield for heavy-ion collisions at the AGS energies and have found that, indeed, the contribution to kaon yield from the process $M M \rightarrow K \bar{K}$ is appreciable. With refined theoretical studies, we hope to extract from the experimental data more definitive information on the properties of hadrons and their interactions in hot and dense matter.

\section{ACKNOWLEDGMENTS}

We are grateful to John Durso for discussions on the cross-section formulas. We thank Berndt Müller for the suggestion that hyperon resonances are important in strangeness equilibration. We also wish to thank Mannque Rho for criticism and participation in many of the conceptual developments. One of the authors (G.E.B.) acknowledges helpful conversations with J. Zimanyi, whose visit was supported by NSF Grant No. INT-821078. The work of G. E. B. was supported in part by the Department of Energy under Grant No. DEFG02-88ER40388 while that of C. M. K., Z. G. W., and L. H. X. was supported in part by the National Science Foundation under Grant No. PHY-8907986 and the Robert A. Welch Foundation under Grant No. A-1110.
${ }^{*}$ Present address: Physics Department, Oregon State University, Corvallis, OR 97331.

${ }^{1}$ H. Stöcker and W. Greiner, Phys. Rep. 137, 278 (1986).

${ }^{2}$ H. Sorge, A. von Keitz, R. Mattiello, H. Stöcker, and W. Greiner, Phys. Lett. B 243, 7 (1990).

${ }^{3}$ M. Gyulassy and W. Greiner, Ann. Phys. (N.Y.) 109, 485 (1977).

${ }^{4}$ G. F. Bertsch, G. E. Brown, V. Koch, and B. A. Li, Nucl. Phys. A490, 745 (1988); G. E. Brown, E. Oset, M. Vicente Vacas, and W. Weise, ibid. A505, 823 (1989).

5J. Q. Wu and C. M. Ko, Nucl. Phys. A499, 810 (1989).

${ }^{6} \mathrm{~L}$. H. Xia and C. M. Ko, in Proceedings of International Workshop XVIII on Gross Properties of Nuclei and Nuclear Excitations, edited by $\mathrm{H}$. Feldmeier (Gesellschaft für Schwerioneuforschung, Darmstadt, Germany, 1990), p. 7.
${ }^{7}$ C. Gale and J. Kapusta, Phys. Rev. C 35, 2107 (1987).

${ }^{8}$ L. H. Xia, C. M. Ko, L. Xiong, and J. Q. Wu, Nucl. Phys. A485, 721 (1988).

${ }^{9}$ C. M. Ko, L. H. Xia, and P. J. Siemens, Phys. Lett. B 231, 16 (1989); C. Korpa, L. Xiong, C. M. Ko, and P. J. Siemens, ibid. 246, 333 (1990).

10J. Randrup and C. M. Ko, Nucl. Phys. A343, 519 (1980); A411, 537 (1983).

${ }^{11}$ J. Aichelin and C. M. Ko, Phys. Rev. Lett. 55, 2661 (1985).

${ }^{12}$ J. Cugnon and R. M. Lombard, Nucl. Phys. A422, 635 (1984).

${ }^{13}$ L. Xiong, C. M. Ko, and J. Q. Wu, Phys. Rev. C 42, 2231 (1990).

${ }^{14}$ T. Abbott et al., Phys. Rev. Lett. 64, 847 (1990).

${ }^{15}$ Y. Miake, in Proceedings of Workshop on Heavy Ion Collisions at the AGS, edited by O. Hauson (Brookhaven National Labo- 
ratory, Upton, New York, 1990), p. 240.

${ }^{16}$ C. M. Ko and L. H. Xia, Phys. Rev. C 38, 179 (1988); Nucl. Phys. A498, 561c (1989); Phys. Lett. B 222, 343 (1989).

${ }^{17}$ H. W. Barz, B. L. Friman, J. Knoll, and H. Shulz, Nucl. Phys. A485, 685 (1988).

18J. Barrett et al., Phys. Rev. Lett. 64, 1219 (1990).

${ }^{19}$ J. Stachel and P. Braun-Munzinger, Phys. Lett. B 216, 1 (1989).

${ }^{20}$ W. Q. Chao, C. S. Gao, and Y. L. Zhu, Nucl. Phys. A514, 734 (1990).

${ }^{21}$ R. Mattiello, H. Sorge, H. Stöcker, and W. Greiner, Phys. Rev. Lett. 63, 1459 (1989).

${ }^{22}$ D. Lohse, J. W. Durso, K. Holinde, and J. Speth, Phys. Lett. B 234, 235 (1990); Nucl. Phys. A516, 513 (1990).

${ }^{23}$ S. D. Protopoescu et al., Phys. Rev. D 7, 1279 (1973).

${ }^{24}$ A. D. Martin and E. N. Ozmutlu, Nucl. Phys. B158, 520 (1977).

${ }^{25}$ D. B. Kaplan and A. E. Nelson, Phys. Lett. B 175, 57 (1986); 179, 409 (1986); see also, A. E. Nelson and D. B. Kaplan, ibid. 192, 193 (1987)

${ }^{26}$ G. E. Brown, Prog. Theor. Phys. 91, 85 (1987).

${ }^{27}$ B. Friedman, V. J. Pandharipanda, and Q. N. Usmani, Nucl. Phys. A372, 483 (1981).

${ }^{28}$ V. Bernard, U. Meissner, and I. Zahed, Phys. Rev. Lett. 59, 966 (1987); V. Bernard and U. G. Meissner, Phys. Lett. B 227, 165 (1989).

${ }^{29}$ G. E. Brown, C. B. Dover, P. B. Siegel, and W. Weise, Phys. Rev. Lett. 60, 2723 (1988).

${ }^{30}$ G. E Brown, A. Sethi, and N. M. Hintz, Phys. Rev. C (to be published).

${ }^{31}$ G. E. Brown and M. Rho, Phys. Lett. B 222, 324 (1989).

${ }^{32}$ T. Hatsuda and T. Kunihiro, Phys. Rev. Lett. 55, 158 (1985); Phys. Lett. B 185, 304 (1987).
${ }^{33}$ R. J. Furnstahl, T. Hatsuda, and S. H. Lee, Phys. Rev. D 42, 1744 (1990); C. Adami, T. Hatsuda, and I. Zahed (unpublished).

${ }^{34}$ G. E. Brown, in Proceedings of the Akito Arima Symposium, Santa Fe, New Mexico, 1990 [Nucl. Phys. A522, 3976 (1991)].

${ }^{35}$ Y. Nambu, in FestiVal-Festschrift for Val Telegdi, edited by K. Winter (Elsevier, New York, 1988).

${ }^{36}$ H. Leutwyler and A. V. Smigla, Nucl. Phys. B342, 302 (1990).

${ }^{37}$ G. E. Brown, K. Kubodera, and M. Rho, Phys. Lett. B 192, 273 (1987).

${ }^{38}$ G. E. Brown, Phys. Rep. 163, 167 (1988).

${ }^{39}$ C. W. Wong, D. Vuong, and K. C. Chu, Nucl. Phys. A515, 686 (1990).

${ }^{40}$ P. Gerber and H. Leutwyler, Nucl. Phys. B321, 387 (1989).

${ }^{41}$ T. S. Birö, H. W. Barz, B. Kukacs, and J. Zimányi, Phys. Rev. C 27, 2695 (1985).

${ }^{42}$ K. S. Lee, M. Rhoades-Brown, and U. Heinz, Phys. Rev. C 37, 1463 (1988).

${ }^{43}$ P. J. Siemens and J. Rasmussen, Phys. Rev. Lett. 42, 844 (1979).

${ }^{44}$ G. E. Brown, J. Stachel, and G. M. Welke, Phys. Lett. B 253, 19 (1991).

${ }^{45}$ C. M. Ko, Phys. Rev. C 23, 2760 (1981).

${ }^{46}$ B. D. Serot and J. D. Walecka, in Advances in Nuclear Physics, edited by J.W. Negele and E. Vogt (Plenum, New York, 1986), Vol. 16.

${ }^{47}$ C. M. Ko, Q. Li, and R. Wang, Phys. Rev. Lett. 59, 1084 (1987); Q. Li and C. M. Ko, Mod. Phys. Lett. A3, 465 (1988); C. M. Ko and Q. Li, Phys. Rev. C 37, 2270 (1988); Q. Li, J. Q. Wu, and C. M. Ko, ibid. 39, 849 (1989); C. M. Ko, Nucl. Phys. A495, 321c (1989).

${ }^{48}$ B. W. Lynn, A. E. Nelson, and N. Tetradis, Nucl. Phys. B345, 186 (1990) 